\title{
PROPHYLACTIC ANTIBIOTICS ALONE VERSUS COMBINED PROPHYLAXIS AND POSTOPERATIVE ANTIBIOTICS FOR PREVENTING SURGICAL-SITE INFECTIONS ASSOCIATED WITH EAR AND NOSE RECONSTRUCTIVE SURGERY
}

\author{
TRIMARTANI KOENTO ${ }^{1}$, RANGGA R. SALEH ${ }^{*}$
}

1Plastic and Reconstructive Division, Department of Otorhinolaryngology Head and Neck Surgery, Cipto Mangunkusumo Hospital-Faculty of Medicine, Universitas Indonesia, Jakarta, Indonesia, ${ }^{2}$ Department of Otorhinolaryngology Head and Neck Surgery, Cipto

Mangunkusumo Hospital-Faculty of Medicine, Universitas Indonesia, Jakarta, Indonesia

Email: rrsaleh@gmail.com

Received: 14 Dec 2018, Revised and Accepted: 10 Mar 2019

ABSTRACT

Objective: The aim of this study was to acquire supporting data for the rational use of antibiotics in plastic reconstructive surgery in the Ear-NoseThroat/Head and Neck Surgery Department at Cipto Mangunkusumo Hospital-Faculty of Medicine Indonesia University.

Methods: This pilot study, with a negative trial design, included 12 subjects who were randomly divided into two groups: preoperative prophylactic antibiotic-only group and a combination of prophylactic and postoperative antibiotic group (controls).

Results: The results showed that only 1 of the 12 subjects (in the prophylactic antibiotic-only group) developed an SSI. None of the controls (combined-use group) had an SSI. The difference between the two groups was not significant.

Conclusion: The use of postoperative antibiotic appears not to be necessary in plastic reconstructive surgery patients to prevent SSIs. Further study, however, is required to support the findings of this study.

Keywords: Ear reconstructive surgery, Nose reconstructive surgery, Postoperative antibiotic, Prophylactic antibiotic, Surgical-site infection

(c) 2019 The Authors. Published by Innovare Academic Sciences Pvt Ltd. This is an open access article under the CC BY license (http://creativecommons. org/licenses/by/4. 0/) DOI: http://dx.doi.org/10.22159/ijap.2019.v11s6.33534

\section{INTRODUCTION}

Surgical-site infections comprise one of the most common causes of morbidity and mortality following head and neck surgery. Infection has a negative effect on the prognosis, prolongs hospitalization, and contributes to a less acceptable cosmetic outcome. The cost of care for patients with infection triples compared with that for patients without infection. The incidence of infection is reported at 8-45\% with the proper use of prophylactic antibiotics. [1-4].

Surgical wound infection is characterized by signs of acute inflammation such as edema, hyperemia, purulent secretion production, and pain, and may be accompanied by fever. Infection of surgical wounds causes disruption of the wound-healing process, which is divided into three phases: inflammation, proliferation, and remodeling [5].

The most common pathogen of SSIs following head and surgery is Staphylococcus aureus. Data from the Ear Nose and Throat (ENT) Department at Cipto Mangunkusumo General Hospital revealed that Klebsiella pneumoniae (16.67\%), Klebsiella oxytoca $(12.5 \%)$, and Staphylococcus epidermidis $(12.5 \%)$ were their most common pathogens. SSIs after head and neck surgery are caused by surgical wound contamination with endogenous flora from the skin or aerodigestive tract. One of the strategies used to reduce the risk of SSIs is decolonization using antiseptic soap [5-8].

The rational use of antibiotics is important for preventing microorganism resistance, decreasing hospital-related infections, and maintaining cost-effectiveness. If infection is diagnosed, it is common to prescribe an empirical antibiotic. The duration of surgery also determines the use of prophylactic antibiotics. Reconstructive plastic surgery in the ENT field is often lengthy. Ear reconstruction, for example, requires a prolonged operation time because of the complexity of the actions taken, starting with rib removal to obtain material with which to forming the framework to then positioning that framework. Prophylactic antibiotics can be given during surgery that lasts $>4 \mathrm{~h}$ by repeating the dose intraoperatively according to the antibiotic's half-life. Existing research suggests that repeated doses of intraoperative cefazolin during surgery lasting $>4 \mathrm{~h}$ reduced the incidence of SSIs from $16.0 \%$ to $7.7 \%$ [1]
The World Health Organization has established antimicrobial resistance as a global health problem. Increased human mobility and world trade result in the possibility of resistance spreading worldwide, with uncontrolled use of antibiotics an important factor in accelerating the rate of antimicrobial resistance [5, 9].

This study generally aimed to obtain data to support the scientific rational use of antibiotics in the management of ear and nose reconstructive surgery in the Department of OtorhinolaryngologyHead and Neck Surgery (ORL-HNS) at the Cipto Mangunkusumo Hospital-Faculty of Medicine Indonesia University (CMH-FMUI). This study specifically aimed to determine the incidence of postoperative infections following ear and nose reconstructive surgery in our department with either prophylactic antibiotic use only or a combination of prophylactic and postoperative antibiotics. We recorded the incidence of edema, hyperemia, pain, and purulent secretion production in SSIs after ear and nose reconstructive surgery to determine the relation between administering a prophylactic antibiotic alone versus combined prophylactic and postoperative antibiotic on the incidence of SSIs following ear and nose reconstructive plastic surgery.

\section{MATERIALS AND METHODS}

This research is a preliminary study in which we planned to enroll 10 consecutive patients per group over a 6-month period. Patients included in this study were male and female, 6-60 y old, who underwent an ear or nose reconstructive procedure in the Otorhinolaryngology Head and Neck Surgery Department Cipto Mangunkusumo Hospital. Patients who had a history of antibiotics use $7 \mathrm{~d}$ prior to surgery or a chronic infection were excluded from the study. The remaining patients were grouped into treatment and control groups based on a randomization method that comprised giving patients envelopes containing numbers. Those with odd numbers were assigned to the treatment group and those with even numbers to the control group. These patients then underwent the following procedures.

1. Surgical preparation that included bathing with an antiseptic solution and shaving. 
2. Nose and/or ear reconstruction surgery.

3. Treatment group were given prophylactic antibiotics alone, whereas the control group were given both prophylactic and postoperative antibiotics.

4. Wound dressings were changed by the researcher, who assessed the wound for signs of infection. Wound care was performed with sterile devices and infection prevention methods (e. g., hand washing) according to CMH standards. Infection prevention methods were used both in the hospital and at home. Evaluation was carried out on postoperative days (PODs) 10, 20, and 30. Variables assessed at each evaluation point indicating a possible SSI were edema, hyperemia, purulent discharge, and postoperative pain. Procalcitonin (a biomarker for postoperative infection) was assayed on POD 7.

5. Patients discharged from the hospital were given an educational sheet regarding at-home wound care.

6. Patients with postoperative infection in either group underwent culture and resistance testing of microorganisms in the surgical wound and were given empirical or definitive antibiotic therapy depending on the resistance evident in the culture examinations. These measures were important for avoiding any ethical issues regarding the management of patients with an SSI.

7. Test results were documented and then analyzed.
Univariate analysis was performed on the subjects' characteristics as middle values and their deviations or frequency distribution. A bivariate analysis was performed to prove the hypothesis by the ${ }^{2}$ test and risk ratio (RR), with $\mathrm{P}<0.05$ indicating significance. The collected data were also processed to determine the control event rate (CER) and the experimental event rate (EER).

\section{RESULTS}

This preliminary research was conducted from February to July 2016. The study initially involved 13 subjects according to the established research criteria, but one subject dropped out during the data collection process.

The subjects with clean or contaminated wounds underwent surgery, with the contaminated clean operations comprising the largest group $(n=7)$. The duration of the surgery in this study had a 4-h cutoff, with more subjects $(n=8)$ undergoing surgery lasting $<4 \mathrm{~h}$. All 12 study subjects received prophylactic antibiotics a maximum of $30 \mathrm{~min}$ before incision, with 4 of them requiring repetition of the prophylactic antibiotic doses because their surgery lasted $\geq 4 \mathrm{~h}$. The prophylactic antibiotic was intravenous cefazolin at a dose of $2 \mathrm{~g}$ in adult patients and a corresponding dose according to body weight in pediatric patients. There were a median of 6 (range 5-10) medical personnel in the operating room during the surgery (table 1).

Table 1: Characteristics of the subjects

\begin{tabular}{ll}
\hline Characteristic & No. of subjects \\
\hline Type of surgery & 2 \\
Stage 1 auiriculoplasty with rib cartilage graft & 1 \\
Stage 2 auriculoplasty with split-thickness skin graft & 1 \\
Otoplasty & 1 \\
Fistulectomy & 2 \\
Septoplasty & 3 \\
Septoplasty with osteotomy & 2 \\
Augmentation rhinoplasty with cartilage graft & 3 \\
Ear reconstruction surgery & 2 \\
With drain & \\
Without drain & 7 \\
Nose reconstruction surgery & 0 \\
With nasal packing & \\
Without nasal packing & \\
\hline
\end{tabular}
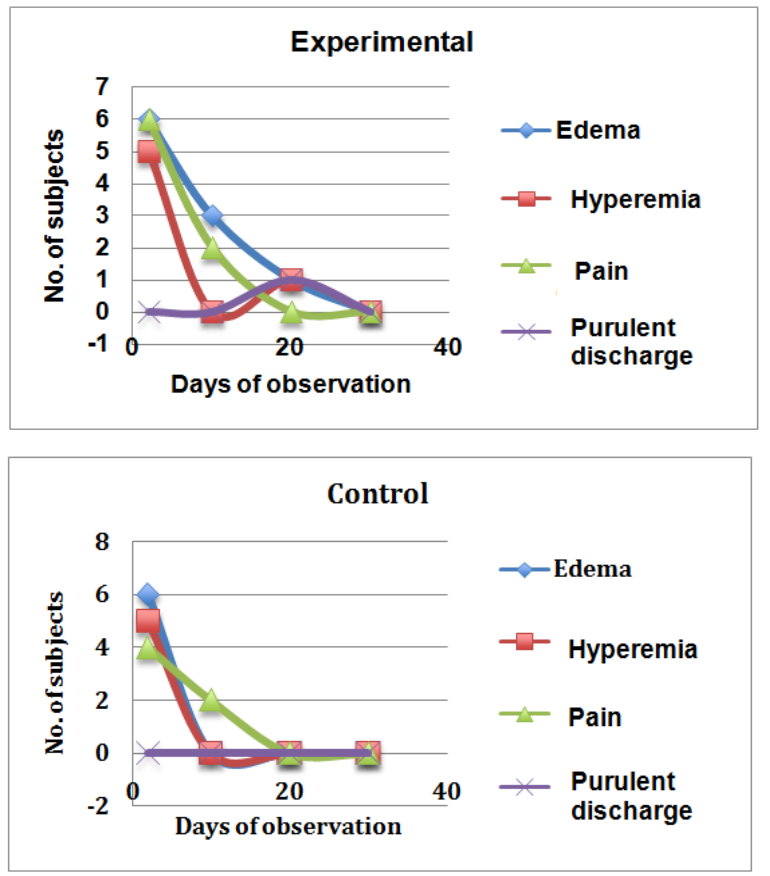

Fig. 1: Postoperative clinical characteristics in the experimental and control groups
Reconstructive surgery varies for both ear and nasal reconstruction. Ear reconstruction ranges from simple actions such as fistulectomy to complex actions such as auriculoplasty with cartilage insertion. All ear reconstruction procedures are performed on skin. Nasal reconstruction is quite different, where there is action concerning the location of the mucosal incision (as for septoplasty) and that in the skin and mucosa (as for augmentation rhinoplasty) with the installation of cartilage grafts.

The incidences of edema, hyperemia, pain, surgical wounds, and purulent discharge in the experimental and control groups from POD 2 to POD 30 are shown in fig. 1. These clinical signs were found mostly on POD 2, decreasing gradually at the assessments on PODs 10, 20, and 30. Diminishing edema was seen more often in the control group than in the experimental group.

Evaluation revealed that only one subject (in the experimental group) still had tissue edema, tissue hyperemia, and purulent secretions by POD 20. Blood procalcitonin and surgical wound swabs were then examined for that patient. The procalcitonin level proved to be elevated $(0.06 \mathrm{mg} / \mathrm{dL})$, and examination of the wound swab revealed the presence of Staphylococcus aureus. Resistance testing showed that the bacteria were sensitive to levofloxacin. Hence, that subject was given levofloxacin $1 \times 500$ mg orally for $5 \mathrm{~d}$. Evaluation on POD 30 showed no signs of edema, hemorrhagic fever, or purulent discharge.

Postoperative clinical features of an SSI include fever, pain at the surgical site, tissue hyperemia, and purulent discharge. At the evaluation on POD 2, most of the study subjects showed signs of 
inflammation in the form of tissue edema $(n=12)$, tissue hyperemia $(n=10)$, and surgical wound pain $(n=10)$. The number of subjects with inflammatory manifestations diminished on PODs 10 and 20, and on POD 30 there was no edema, hyperemia, surgical wound pain, or purulent discharge in any of the subjects. The relation between the clinical parameters (tissue edema, tissue hyperemia, purulent discharge, surgical wound pain) and the possibility of an SSI is shown in table 2 .

Table 2: Clinical features and surgical-site infections

\begin{tabular}{|c|c|c|c|}
\hline \multirow[t]{2}{*}{ Variable } & \multicolumn{2}{|c|}{ Surgical-site infection } & \multirow[t]{2}{*}{$\mathbf{P}$} \\
\hline & Yes $(n=1)$ & No $(n=11)$ & \\
\hline \multicolumn{4}{|l|}{ Pain } \\
\hline POD $2(n=10)$ & 1 & 9 & 1.000 \\
\hline POD $10(n=4)$ & 0 & 4 & 1.000 \\
\hline \multicolumn{4}{|l|}{ Edema } \\
\hline POD $10(n=3)$ & 0 & 3 & 1.000 \\
\hline POD $20(n=1)$ & 1 & 0 & 0.083 \\
\hline \multicolumn{4}{|l|}{ Hyperemia } \\
\hline POD $2(n=10)$ & 0 & 10 & 0.167 \\
\hline POD $20(n=1)$ & 1 & 0 & 0.083 \\
\hline \multicolumn{4}{|l|}{ Purulent discharge } \\
\hline POD $20(n=1)$ & 1 & 0 & 0.083 \\
\hline
\end{tabular}

POD, postoperative day

Table 3: Relation between prophylactic antibiotics and combined prophylactic and postoperative antibiotics with surgical-site infection

\begin{tabular}{|c|c|c|c|}
\hline \multirow[t]{2}{*}{ Group } & \multicolumn{2}{|c|}{ Surgical-site infection } & \multirow[t]{2}{*}{$\mathbf{P}$} \\
\hline & Yes $(n=1)$ & No $(n=11)$ & \\
\hline Prophylactic antibiotic+postoperative antibiotic & 0 & 6 & \\
\hline Prophylactic antibiotic only & 1 & 5 & 1.000 \\
\hline
\end{tabular}

The relation between prophylactic antibiotic administration versus a combination of prophylactic antibiotics and postoperative antibiotics with the incidence of SSIs is shown in table 3. One subject in the prophylactic antibiotic (experimental) group experienced an SSI, whereas there were no SSIs in the combined prophylactic antibiotic and postoperative antibiotic group. There was no significant difference between the experimental and control groups $(p=1.000)$. The CER, EER, absolute risk reduction (ARR) and number needed to treat (NNT) were calculated from the data in table 3 . In this study, CER 0, EER 0.167 , ARR -0.167 , and NNT -6 were obtained.

\section{DISCUSSION}

The types of surgical wounds in these research subjects were clean and clean-contaminated. This type of surgery can also affect the incidence of SSIs as the risk increases with the degree of contamination of the incision. In this study, subjects with SSIs were included in the type of contaminated-clean surgery. In addition to the type of wound, the location of the incision affectd the risk of an SSI. There are differences in wound healing of skin and mucosa. Mucosal wounds heal faster and with less scarring than skin lesions-perhaps because of the greater hypoxic condition in the skin than in the mucosa, although oxygen is important for optimal wound healing. This hypoxic state is characterized by higher HIF-1 $\alpha$ expression in skin lesions than mucosal lesions $[5,10]$.

A prolonged operating duration can increase the risk of an SSI. Based on Nwankwo et al., the operating duration of $>2 \mathrm{~h}$ can increase the risk of surgical wound infection fourfold. This study used a 4-h limit to assess the duration of surgery (based on other studies). In this study, the incidence of surgical wound infection occurred in 1 subject with an operating duration of $<4 \mathrm{~h}[5,11]$.

All study subjects received a prophylactic antibiotic (i.e., intravenous cefazolin). The choice of cefazolin as a prophylactic antibiotic is based on the prevailing resistance pattern at $\mathrm{CMH}$. Cefazolin is a first-generation, broad-spectrum cephalosporin antibiotic, effective against Gram-positive and Gram-negative bacteria. The dose of cefazolin for prophylaxis in adults is $2 \mathrm{~g}$ intravenously. In children, the dose is measured as $30 \mathrm{mg} / \mathrm{kg}$ body weight per dose $[12,13]$.

All subjects in this study received prophylactic antibiotics within 30 min before the incision. Based on the literature, the optimal time to provide a prophylactic antibiotic is $0-30 \mathrm{~min}$ before the incision. The risk of infection is increased if the prophylactic antibiotic is administered $>30$ min before or after the incision. Prophylactic antibiotic doses can be repeated if the duration of surgery is $>4 \mathrm{~h}$. In this study, four subjects underwent with an operating that lasted $\geq 4$ h. All subjects received repeated prophylactic antibiotic doses at 4-h intervals $[5,13,14]$.

The number of medical personnel in the operating room can also affect the incidence of SSIs. Nwankwo et al. found a significant difference in the incidence of SSIs when there were more than six medical personnel present. In this study, an SSI occurred in one subject whose intraoperative staff included six medical personnel. The highest number of intraoperative medical personnel was 10 in this study, but with no other subject developing an SSI $[11,14]$.

Drains are often placed during ear reconstructive surgery. Three of our research subjects required drainage. The drain could increase the risk of SSIs because it is an entry route for bacteria. Therefore, it must be removed as quickly as possible. In two subjects who underwent stage 1 auriculoplasty, the drain was removed on POD 5, whereas in those who underwent fistulectomy they were removed on POD 2. This difference is due to the function of the flow following these two operations. For auriculoplasty, in addition to drainage, saliva functions as a negative pressure provider to achieve better aesthetic results $[5,15,16]$.

Among our 12 subjects, 7 required anterior nasal packing, which could be a source of bacterial infection. In addition, the installation of nasal packing poses a risk of toxic shock syndrome due to infection with Staphylococcus aureus or Streptococcus pyogenes. Routine antibiotics are used after posterior packing, such as coamoxiclav $625 \mathrm{mg}$, up to three doses per day starting from the time of drainage installation until up to $5 \mathrm{~d}$ after its removal. For anterior packing, a systemic antibiotic is not required if the packing is removed within $48 \mathrm{~h}$. If the packing is maintained for $>48 \mathrm{~h}$, coamoxiclav $625 \mathrm{mg}$ is given three times per day for up to $5 \mathrm{~d}$ after the packing is removed. In this study, seven subjects with postoperative anterior packing underwent its removal $48 \mathrm{~h}$ later, so there was no need for a systemic antibiotic. The principle of preventing infection due to nasal packing is applicable not only to postoperative patients but also those with epistaxis [17]. 
At the POD 20 evaluation, only 1 of the 12 subjects (from the experimental group) experienced edema, hyperemia, and purulent discharge as well as an elevated procalcitonin level $(0.06 \mathrm{mg} \mathrm{dL})$ when compared with that on POD $7(0.02 \mathrm{mg} / \mathrm{dL})$. The presence of those clinical features accompanied by an increased procalcitonin level led to the diagnosis of an SSI. The subjects diagnosed with nasal os fractures underwent septorhinoplasty with osteotomy. On POD 20 , they had septal edema and hyperemia that had not been present at the previous evaluation. In addition, there were purulent secretions originating from the media meatus so it was suspected that there was an SSI that manifested as acute sinusitis accompanied by septal cellulitis. Septorhinoplasty is considered cleancontaminated surgery, and bacterial flora and normal infections can occur in the nasal mucosa. The most common cause of an SSI following septorhinoplasty is Staphylococcus aureus. This study obtained similar results, with the microorganisms cultured from septal swabs and media meatus were identified as $S$. aureus. Andrews reported the most infectious bacterium, S. aureus, in seven subjects. Other pathogenic bacteria include Klebsiella sp. and Proteus $\mathrm{sp}$, Salmonella, and Morganella morganii [5, 18].

The affected subject in this preliminary trial was given definitive antibiotic therapy based on the results of resistance cultures. Staphylococcus aureus, the pathogenic bacterium in our subject, was sensitive to amoxicillin-clavulanate. However, because many individuals are allergic to amoxicillin, other antibiotics have been adopted, including levofloxacin, to which this $S$. aureus was sensitive. This subject was given levofloxacin for $5 \mathrm{~d}$, at which time the edema, hyperemia, and purulent secretions had disappeared. Levofloxacin, a respiratory fluoroquinolone, is recommended as an alternative in patients with acute sinusitis with penicillin allergy. The duration of antibiotic administration is determined by the clinical response. According to a systematic literature review of antibiotic use in bacterial acute sinusitis, data indicated that administration of antibiotics for $5 \mathrm{~d}$ had the same effectiveness as when given for $10 \mathrm{~d}$. The duration of long-term antibiotic administration is related to the appearance of drug-related side effects. The incidence of these side effects was lower in patients with a 5-day regimen than in those on a 10 -day regimen, with an odds ratio of $0.79[19,20]$.

SSIs are caused by various factors. In our subject, the infection manifested as edema and hyperemia of the septal mucosa accompanied by a purulent discharge from the media meatus. These clinical features indicated the presence of acute sinusitis accompanied by septal cellulitis. Based on the literature, complications of an infection after septorhinoplasty can be acute or chronic sinusitis, caused by disruption of the function of the osteomeatal complex due to actions taken during septorhinoplasty (e. g., osteotomy). Andrews et al. reported two subjects who experienced septal cellulitis complications that were included in the criteria for "major" infection. The study classified the degree of infection of the surgical wound as a minor degree of vestibulitis or a major degree of septal or nasal cellulitis and secondary bleeding. The infection that occurred in our research subject might have been influenced by occupational factors. The subject with the SSI was a nurse at $\mathrm{CMH}$. Based on the epidemiological triad (host-agentenvironment), infections could be due to the interaction of the three factors. This subject, as a nurse, had repeated contact with sources of infection $[18,21,22]$.

In the present study, the number of subjects with inflammatory features in the form of edema, hyperemia, and surgical wound pain continued to decreased on PODs 10 and 20 until, finally, on POD 30, no clinical manifestations were found in any of the subjects. This is consistent with the wound-healing phase, where during the proliferative and remodeling phases, the role of inflammatory cytokines has begun to diminish and is being replaced by a fibrin matrix and granulation tissue. The remodeling phase, however, continues for about a year $[23,24]$.

In this study, the postoperative clinical features were associated with the incidence of SSIs. In the assessment of surgical wound pain on PODs 2 and 10 , there was no significant association with an SSI $(\mathrm{p}=1.000)$. Evaluation of edema on PODs 10 and 20 also found no significant relation $(p=1.000$ and $p=0.083$, respectively). Likewise with hyperemic assessment on PODs 2 and $20(p=0.167$ and $p=0.083$, respectively $)$ and purulent discharge on POD $20(\mathrm{p}=0.083)$. However, the presence of edema, hyperemia, and purulent discharge on POD 20 could be an indication of SSI. This is in accordance with the process of wound healing, wherein the signs of inflammation have begun to decrease at the end of the inflammatory phase (POD 7). The presence of inflammatory signs on POD 20 indicates the possibility of an infection that interferes with the healing process of the wound $[5,23,24]$.

The incidence of SSIs in the current study included only one subject in the experimental group. None of the subjects in the control group had an SSI. The proportional difference in these two groups was not statistically significant $(p=1.000)$. This finding is consistent with a study, conducted by Andrews et al., which found that there were no significant differences between administering prophylactic and postoperative antibiotics for septorhinoplasty surgery. Andrews et al. reported the proportion of SSIs in the prophylactic antibiotic group at $7 \%$ and in the postoperative antibiotic group at $11 \%$, with the total proportion of SSIs for all subjects at 9\%. The present study, though, had differences in patient selection. Our study involved those who underwent clean and clean-contaminated surgery, whereas that of Andrews et al. involved only subjects who underwent clean-contaminated surgery [18].

Pathogenic bacteria that caused the infection in this study was Staphylococcus aureus, which has an incubation period of 2-10 d. In subjects with SSIs, signs of infection are usually apparent by POD 20. Based on these findings, it can be concluded that bacterial infections manifest on PODs 10-18. The postoperative antibiotic in this study was administered on POD 7. Therefore, the risk of infection in these subjects was no different between those given a prophylactic antibiotic only and those given a prophylactic antibiotic and a postoperative antibiotic [25]

Statistical calculations showed EER values of 0.167 , CER 0, ARR -0.167 , and NNT -6 . The ARR value was $<0$, so it can be concluded that the absolute risk increased in the experimental group. In this study, the absolute risk of developing an SSI after a prophylactic antibiotic alone increased by $16.7 \%$ when compared with those given the prophylactic antibiotic-postoperative antibiotic combination. The NNT was also $<0$ so it was concluded that it was the number needed to harm (not to treat). Thus, in this study, the number to harm was six, so it can be concluded that when giving only six patients a prophylactic antibiotic, one patient would develop an SSI.

This research comprises a preliminary study with a design that aims to prove the differences in proportions between two groups. It did prove that the proportion of the two groups was not significantly different, but it needs to be continued in a negative clinical trial that requires a larger sample size. The present study found one SSI in the prophylactic antibiotic-only group. However, this study was not able to eliminate the confounding factors that exist primarily from environmental factors as the cause of SSIs.

\section{CONCLUSION}

The rational use of perioperative antibiotics is important for decreasing the risk of SSIs. Although there was no significant relation found in this study, further evaluation is required to prove the effectiveness of the rational use of antibiotics for avoiding SSIs. Such infections can be diagnosed via a thorough postoperative examination looking for typical signs such as edema, hyperemia, and purulent discharge.

\section{ACKNOWLEDGEMENT}

This paper was presented at The $3^{\text {rd }}$ International Conference and Exhibition on Indonesian Medical Education and Research Institute (ICE on IMERI 2018), Faculty of Medicine, Universitas Indonesia, Jakarta. We thank the $3^{\text {rd }}$ ICE on IMERI Committee who had supported the peer review and manuscript preparation before submitting to the journal.

\section{AUTHORS' CONTRIBUTIONS}

The first author of this study performed the surgery on the subjects, and the second author was responsible for their postoperative care. Both authors evaluated the preoperative, intraoperative, and postoperative findings that comprise the results. 


\section{CONFLICTS OF INTERESTS}

All authors have none to declare

\section{REFERENCES}

1. Salkind AR, Rao KC. Antibiotic prophylaxis to prevent surgical site infections. Am Fam Physician 2011;83:585-90.

2. Uçkay I, Harbarth S, Peter R, Lew D, Hoffmeyer P, Pittet D. Preventing surgical site infections. Expert Rev Anti Infect Ther 2010;8:657-70.

3. Saxena A, Singh MP, Brahmchari S, Banerjee M. Surgical site infection among postoperative patients of tertiary care centre in Central India: a prospective study. Asian J Biomed Pharm Sci 2013;3:41-4.

4. Spratt D, Dutton RP, Dellinger EP, Bird B, Guglielmi CL, Farber $\mathrm{M}$, et al. The role of the health care professions in preventing surgical site infection. AORN J 2012;95:430-40.

5. Rosenberger LH, Sawyer RG. Surgical site infections. In: Cameron JL, Cameron AM. editors. In: Current Surgical Therapy. 11th Edition. 11th ed. Elsevier; 2014. p. 1172-7.

6. Noorani A, Rabey N, Walsh SR, Davies RJ. Systematic review and meta-analysis of preoperative antisepsis with chlorhexidine versus povidone-iodine in clean-contaminated surgery. Br J Surg 2010;97:1614-20.

7. Darouiche RO, Wall MJ Jr, Itani KM, Otterson MF, Webb AL Carrick MM, et al. Chlorhexidine-alcohol versus povidone-iodine for surgical-site antisepsis. N Engl J Med 2010;362:18-26.

8. Loho T, Astrawinata DA. Bacterial and antibiotics susceptibility profile at cipto mangunkusumo general hospital Jan-Jun 2015. 1st edition. Loho T. editor. Jakarta: Division of Infectious Disease, Department of Clinical Pathology Cipto Mangunkusumo General Hospital; 2015. p. 171-5.

9. Van Boeckel TP, Gandra S, Ashok A, Caudron Q, Grenfell BT, Levin SA, et al. Global antibiotic consumption 2000 to 2010:an analysis of national pharmaceutical sales data. Lancet Infect Dis 2014;14:742-50.

10. Chen L, Gajendrareddy PK, DiPietro LA. Differential expression of HIF-1 $\alpha$ in skin and mucosal wounds. J Dent Res 2012;91:871-6.

11. Nwankwo EO, Ibeh I, Enabulele OI. Incidence and risk factors of surgical site infection in a tertiary health institution in Kano, northwestern Nigeria. Int J Infect Control 2012;8:8-13.

12. Bratzler DW, Dellinger EP, Olsen KM, Perl TM, Auwaerter PG, Bolon MK, et al. Clinical practice guidelines for antimicrobial prophylaxis in surgery. Am J Heal Pharm 2013;70:195-283.

13. Shankar RP, Partha P, Shenoy NK, Easow JM, Brahmadathan KN. Prescribing patterns of antibiotics and sensitivity patterns of common microorganisms in the internal medicine ward of a teaching hospital in Western Nepal: a prospective study. Ann Clin Microbiol Antimicrob 2003;2:7.

14. Collier M, E Ans D Vid, Farrington M, Gibbs E, Gould K, Kathryn $\mathrm{HJ}$, et al. Surgical site infection: prevention and treatment of surgical site infection. In: National Institute for Health and Care Excellence-Clinical Guidelines [Internet]; 2008. p. 168.

15. Murakami C, Quatela V, Sie K, Shyidler J. Microtia reconstruction. In: Flint PW, Haughey BH, Lund VJ, Richardson MA, Robbins KT, Thomas JR, et al. editors. Cummings Otolaryngology Head and Neck Surgery. 5th ed. Philladelphia: Mosby Elsevier; 2010. p. 2741-51.

16. Kelley PE, Scholes MA. Microtia and congenital aural atresia. Otolaryngol Clin North Am 2007;40:61-80.

17. Biggs TC, Nightingale K, Patel NN, Salib RJ. Should prophylactic antibiotics be used routinely in epistaxis patients with nasal packs? Ann R Coll Surg Engl 2013;95:40-2.

18. Andrews PJ, East CA, Jayaraj SM, Badia L, Panagamuwa C, Harding L. Prophylactic vs postoperative antibiotic use in complex septorhinoplasty surgery: a prospective, randomized, single-blind trial comparing efficacy. Arch Facial Plast Surg 2006;8:84-7.

19. Rosenfeld RM, Piccirillo JF, Chandrasekhar SS, Brook I, Ashok Kumar K, Kramper M, et al. Clinical practice guideline (update): adult sinusitis. Otolaryngol Head Neck Surg 2015;152(Suppl):S139.

20. Stevens DL, Bisno AL, Chambers HF, Everett D, Dellinger P, Goldstein EJC, et al. Practice guidelines for the diagnosis and management of skin and soft-tissue infections. Clin Infect Dis 2005;41:1373-406.

21. Daniel RK. A basic rhinoplasty operation. In: Mastering rhinoplasty a comprehensive atlas of surgical techniques with integrated video clips. Springer; 2010. p. 13-66.

22. Pittet D. Hand hygiene in health care first global patient safety challenge clean care is safer care. World Health 2009;30:270.

23. Gurtner GC. Wound healing: normal and abnormal. In: Thorne C. editor. Grabb and Smith's Plastic Surgery. 6th edition. Lippincott Williams and Wilkins; Philadelphia; 2007. p. 1522.

24. Verwoerd-Verhoef HL, Koppel PG, Osch GJ van, Meeuwis CA, Verwoerd C DA. Wound healing and cartilage structures in the head and neck region. Wound Heal Distortion Gener Cartil 2005; 43:17-30.

25. Russel EJ, Czarvionke L, Huckman M, Daniels D, McLachlan D. CT of the inferomedial orbit and the lacrimal drainage apparatus: normal and pathologic anatomy. Am J Roentgenol 1985;145:1147-54. 